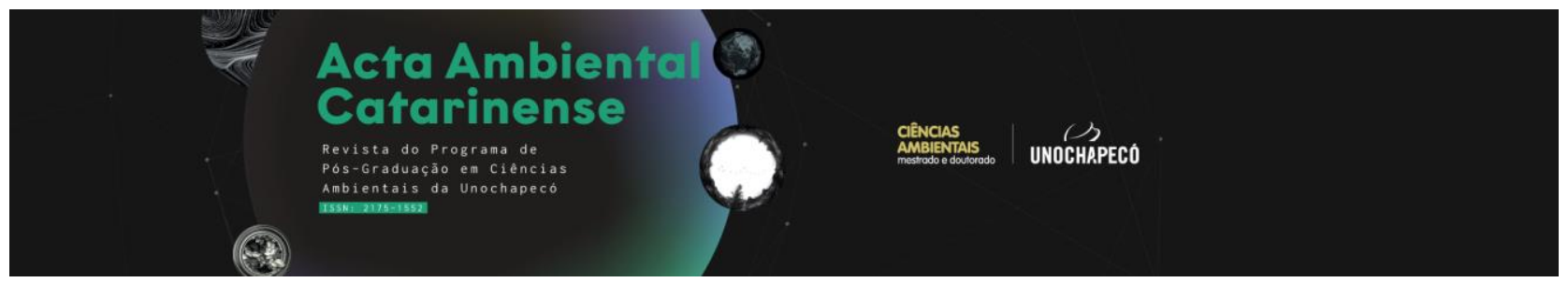

\title{
LEVANTAMENTO DA FERTILIDADE DO SOLO DE PROPRIEDADES RURAIS DO MUNÍCIPIO DE CHAPECÓ - SC: UM ESTUDO DE CASO
}

\author{
Alana Maria Polesso ${ }^{1}$ \\ Evandro Spagnollo ${ }^{2}$ \\ Carolina Riviera Duarte Maluche Baretta ${ }^{3}$
}

\begin{abstract}
Resumo
O manejo da fertilidade do solo é a base para a busca do aumento da produtividade e inclui a análise de solo como medida para correção da acidez e, posteriormente, aplicações de fertilizantes. $\mathrm{O}$ estudo objetivou caracterizar a fertilidade média de áreas rurais do município de Chapecó - SC, a partir do banco de dados de análises realizadas nos anos de 2016 e 2017 fornecido pelo Laboratório de Solos da Epagri - Chapecó. Foram avaliados 147 laudos e interpretados os seguintes atributos químicos do solo: $\mathrm{pH}$ - água, saturação por alumínio (m\%), capacidade de troca de cátions a pH 7,0 $\left(\mathrm{CTC}_{\mathrm{pH} 7,0}\right)$, fósforo $(\mathrm{P})$, potássio $(\mathrm{K})$, matéria orgânica do solo $(\mathrm{MOS})$, cálcio $(\mathrm{Ca})$, magnésio $(\mathrm{Mg})$ e saturação por bases $(\mathrm{V} \%)$. Os teores dos nutrientes foram interpretados segundo suas classes de disponibilidade conforme o Manual de Calagem e Adubação para os estados do Rio Grande do Sul e Santa Catarina, e analisados por estatística descritiva. A maior frequência de $\mathrm{pH}$ ocorreu para valores menores que 5,5 (53\%) e entre 5,5 e 5,9 (30\%). Para a V\% das amostras 9\% dos laudos de solo apresentaram valores $\geq 85 \%, 47 \%$ valores de $65-74 \%, 39 \%$ valores de 75 a $84 \%$; e apenas $5 \%$ destes valores $<65 \%$. Praticamente $50 \%$ dos laudos das classes texturais 1,2 e 3 apresentam teores muito alto a alto de P; com $46 \%$ dos laudos com teores muito baixo e $21 \%$ baixo de $\mathrm{P}$ na classe textural 4. Para a $\mathrm{CTC}_{\mathrm{pH}} 7,069 \%$ dos laudos tiveram CTC média $\left(7,6-15 \mathrm{cmol}_{\mathrm{c}} \mathrm{dm}^{-3}\right) \mathrm{com} 100 \%$ destas amostras na classe de disponibilidade muito baixo. Nas demais CTCs encontradas a maior frequência dos valores de $\mathrm{K}$ ocorreu nas faixas muito alto e alto. Os teores de $\mathrm{Ca}$ e $\mathrm{Mg}$ tiveram frequência alta (86 e 93\%, respectivamente). A MOS apresentou em 93\% dos laudos teor médio. Os resultados mostram a necessidade de um cuidado por parte dos produtores e assistência técnica para adequação das doses de nutrientes como o $\mathrm{P}, \mathrm{Ca}$ e $\mathrm{Mg}$; bem como um cuidado quanto a correção do $\mathrm{pH}$ e aumento dos níveis de $\mathrm{K}$ em grande parte dos laudos analisados.
\end{abstract}

Palavras-chave: Análise de solo, Caracterização química, Adubação.

\footnotetext{
${ }^{1}$ Universidade Comunitária da Região de Chapecó - Unochapecó

${ }^{2}$ Empresa de Pesquisa Agropecuária e Extensão Rural de Santa Catarina - EPAGRI

${ }^{3}$ Universidade Comunitária da Região de Chapecó - Unochapecó, Dra. Agronomia 


\begin{abstract}
The management of soil fertility is the basis for the search for increased productivity and includes soil analysis as a measure to correct acidity and, subsequently, fertilizer applications. The study aimed to characterize the average fertility of rural areas in the municipality of Chapecó - SC, from the database of analyzes performed in the years 2016 and 2017 provided by the Soil Laboratory of Epagri-Chapecó. Were evaluated 147 reports and the following soil chemical attributes were interpreted: $\mathrm{pH}$-water, aluminum saturation $(\mathrm{m} \%)$, cation exchange capacity at $\mathrm{pH} 7.0\left(\mathrm{CTC}_{\mathrm{pH} 7.0)}\right)$, phosphorus $(\mathrm{P})$, potassium $(\mathrm{K})$, soil organic matter (MOS), calcium $(\mathrm{Ca})$, magnesium $(\mathrm{Mg})$, and base saturation $(\mathrm{V} \%)$. The nutrient contents were interpreted according to their availability classes based on to the Lime and Fertilization Manual for the states of Rio Grande do Sul and Santa Catarina, and analyzed using descriptive statistics. The highest $\mathrm{pH}$ frequency occurred for values less than 5.5 $(53 \%)$ and between 5.5 and $5.9(30 \%)$. For the V\% of the samples, $9 \%$ of the soil reports had values $\geq 85 \%, 47 \%$ values $65-74 \%, 39 \%$ values $75-84 \%$; and only $5 \%$ of these values $<65 \%$.Practically $50 \%$ of the reports of textural classes 1,2 and 3 have remarkably high to high levels of $\mathrm{P}$, with $46 \%$ of the reports with very low levels and $21 \%$ low of P in textural class 4 . For CTC pH $7.069 \%$ of the reports had average CTC $\left(7.6-15 \mathrm{cmol}_{\mathrm{C}} \mathrm{dm}^{-3}\right)$ with $100 \%$ of these samples in very low availability class. In the other CTCs found, the highest frequency of $\mathrm{K}$ values occurred in the very high and high ranges. The contents of $\mathrm{Ca}$ and $\mathrm{Mg}$ had high frequency (86 and 93\%, respectively). MOS had an average content in $93 \%$ of the reports. The results show the producers need for care more about these levels, using technical assistance to adjust the doses of nutrients such as $\mathrm{P}, \mathrm{Ca}$ and $\mathrm{Mg}$, as well to care about $\mathrm{pH}$ correction and increase in $\mathrm{K}$ levels in most of the analyzed reports.
\end{abstract}

Keywords: Soil analysis, Chemical characterization, Fertilization.

\section{INTRODUÇÃ̃o}

O desenvolvimento de uma agricultura mais tecnológica e ambientalmente sustentável integra um conjunto de práticas e ações que visam o fator humano, social e ambiental, e o correto equilíbrio da promoção da melhoria e/ou manutenção da qualidade física, química e biológica do solo, para promoção da produtividade agrícola (CREPALDI et al., 2014). A correção do solo e doses recomendáveis de nutrientes, juntamente com o avanço do melhoramento genético de plantas foram incluídos no manejo integrado das culturas, o qual considerou também o monitoramento e o manejo da fitossanidade, o controle das plantas daninhas, da irrigação, da colheita e comercialização (BALDOTTO et al., 2015).

Nesse contexto, a amostragem do solo para fins de determinação de sua fertilidade é um dos fatores chaves para determinar os resultados de produção esperados pelos produtores. As condições químicas apresentadas nos laudos das análises de solo têm por princípio determinar os teores dos nutrientes disponíveis no solo e permitem a tomada de decisão quanto a necessidade de sua reposição, escolhendo a forma e o período mais adequado para disponibilização dos nutrientes para a planta (PREZOTTI e GUARÇONI, 2013).

O município de Chapecó - SC está localizado na macrorregião do Oeste Catarinense e apresenta, segundo dados preliminares divulgados pelo Instituto Brasileiro de Geografia e Estatística (IBGE, 2018) 1.661 propriedades rurais, com ocupação de parte de seu solo com vegetação arbórea nativa ou introduzida em áreas agricultáveis com pastagens, culturas anuais e perenes. Como principais atividades agropecuárias destaca-se a produção de ovos, suínos, soja, frango, leite e milho (PREFEITURA MUNICIPAL DE CHAPECÓ, 2015). Com uma topografia 
predominantemente forte ondulada a ondulada é reportada pouca variabilidade dos tipos de solo encontrados no município que, segundo dados do Plano de Desenvolvimento Rural da Prefeitura de Chapecó (2015), incluem os Neossolos Litólicos, Latossolos Brunos e, em menor ocorrência os Cambissolos Háplicos, todos de natureza ácida e de baixa fertilidade natural. Como característica de uso e manejo dos solos da região e do município, aproximadamente 50\% das áreas sob terrenos suavemente ondulados a ondulados encontram-se sob uso de pastagens e plantas anuais, assim como as áreas de maior declividade caracterizam-se por quase $50 \%$ do seu uso para pastagens, culturas anuais, perenes e reflorestamento, tendo como principal sistema de manejo do solo para os cultivos agrícolas o Plantio Direto (SECRETARIA DE ESTADO DO PLANEJAMENTO, ORÇAMENTO E GESTÃO, 2003).

Neste município, a Empresa de Pesquisa Agropecuária e Extensão Rural de Santa Catarina (Epagri), vinculada ao Governo do Estado de Santa Catarina através do seu Centro de Pesquisa para Agricultura Familiar (CEPAF) processa em seu Laboratório de Solos uma média de 28.000 amostras por ano sendo que deste total, aproximadamente $88 \%$ são oriundas de propriedades rurais (EPAGRI/CEPAF, 2018).Os resultados obtidos através dessas análises capacitam o produtor a fim de tornar as atividades desenvolvidas no meio rural mais competitivas e lucrativas, destacando Santa Catarina como referência nacional na produção de alimentos. Além disso, o diagnóstico da fertilidade atual das áreas agrícolas pode auxiliar na prática de extensão rural, melhorando a assistência técnica do produtor do Oeste Catarinense, e dentre eles, o pequeno produtor rural chapecoense, perfil dominante na atividade agropecuária do município. Dessa forma, o presente trabalho teve como objetivo caracterizar a fertilidade média do solo de propriedades rurais do município de Chapecó - SC, a partir do banco de dados de análise realizadas nos anos de 2016-2017, fornecido pelo Laboratório de Solos da Epagri de Chapecó.

\section{MATERIAL E MÉtodos}

\subsection{Obtenção e abrangência dos dados}

A pesquisa utilizou dados coletados a partir das análises de solos de produtores rurais do município de Chapecó - SC, obtidas juntamente com a Empresa de Pesquisa Agropecuária e Extensão Rural de Santa Catarina (Epagri) - Centro de Pesquisa para Agricultura Familiar (CEPAF) do mesmo município. $\mathrm{O}$ município está localizado a uma latitude $27^{\circ} 05^{\prime} 47^{\prime \prime} \mathrm{S}$ e uma longitude de $52^{\circ} 37^{\prime} 06^{\prime \prime} \mathrm{O}$, a $674 \mathrm{~m}$ de altitude e apresenta clima mesotérmico úmido segundo a classificação de Köppen (1918), com verão longo e inverno curto e ameno, com temperaturas médias variando de 11 a $28{ }^{\circ} \mathrm{C}$.

Os laudos de análise foram obtidos do banco de dados do sistema Glebas com resultados numéricos das análises químicas e granulométricas do solo realizadas pela Epagri no período de 2016 a 2017. O levantamento foi realizado abrangendo 147 laudos de solos de todas as propriedades rurais do município de Chapecó que encaminharam amostras para o Laboratório de Solos durante o período. Quando da ocorrência de mais de uma amostra encaminhada por propriedade realizou-se a aleatorização dos laudos, utilizando apenas um laudo por propriedade. Os laudos não apresentam identificação do produtor e da sua respectiva propriedade de origem, não havendo informações sobre qual cultura e/ou atividade destina-se o uso do solo nas propriedades, bem como informações sobre o manejo dos solos, que para o presente estudo basearam-se no histórico agrícola do município obtido a partir do seu Plano de Desenvolvimento Rural (PREFEITURA MUNICIPAL DE CHAPECÓ, 2015). 


\subsection{Método de interpretação dos dados}

Para estabelecer as classes de disponibilidade dos nutrientes no solo foram utilizados os seguintes parâmetros: classificação do solo quanto ao seu conteúdo de argila, $\mathrm{pH}$ : água (1:1), teores de fósforo $(\mathrm{P})$, potássio $(\mathrm{K})$, cálcio $(\mathrm{Ca})$, magnésio $(\mathrm{Mg})$, alumínio (Al) e matéria orgânica do solo (MOS), capacidade de troca de cátions a $\mathrm{pH}$ $7,0\left(\mathrm{CTC}_{\mathrm{pH} 7,0}\right)$, saturação por bases $(\mathrm{V} \%)$ e por alumínio $(\mathrm{m} \%)$. Para interpretação dos laudos foram utilizados os níveis de exigência conforme o grupo 2 do Manual de Calagem e Adubação para os estados do Rio Grande do Sul e Santa Catarina (COMISSÃO DE QUÍMICA E FERTILIDADE DO SOLO $\mathrm{RS} / \mathrm{SC}$, 2016), que considera as seguintes culturas: grãos (exceto arroz irrigado), hortaliças (exceto culturas do grupo 1) e pastagens (exceto pastagem natural), frutíferas e gengibre.

Os valores de $\mathrm{pH}$ foram classificados conforme as faixas $\leq 5,5 ; 5,5-5,9 ; 6,0-6,4$; e $\geq 6,5$. O valor de $m \%$ foi agrupado nas faixas $<5 ; \quad 5-10 ; \quad 10,1-20 ; \quad 20,1-45$ e $>45$, correspondentes às classes muito baixo, baixo, médio, alto e muito alto, segundo Osaki (1991). Os valores de V\% foram agrupados nas faixas $<65 \% ; 65-74 \% ; 75-84 \% \mathrm{e} \geq 85 \%$. A $\mathrm{CTC}_{\mathrm{pH} 7,0}$ do solo $\left(\mathrm{cmol}_{\mathrm{c}} \mathrm{dm}^{-3}\right)$ foi agrupada nas faixas $\leq 7,5 ; 7,6-15,0 ; 15,1-30,0 \mathrm{e}>30,0$ correspondentes as classes baixa, média, alta e muito alta. Para os teores de $\mathrm{P}\left(\mathrm{mg} \mathrm{dm}^{-3}\right)$ os laudos foram inicialmente classificadas de acordo com o teor de argila nas classes 1 (>60\%); classe $2(60-41 \%)$, classe $3(40-21 \%)$ e classe $4(\leq 20 \%)$, sendo posteriormente interpretados nas classes de disponibilidade do nutriente muito baixo, baixo, médio, alto e muito alto. Para a classificação do teor de K $\left(\mathrm{mg} \mathrm{dm}^{-3}\right)$, considerou-se inicialmente a faixa de interpretação para a $\mathrm{CTC}_{\mathrm{pH} 7,0}$ do solo, sendo posteriormente classificado em muito baixo, baixo, médio, alto e muito alto dentro de cada faixa de CTC. O teor de $\mathrm{Ca}\left(\mathrm{cmol}_{\mathrm{c}} \mathrm{dm}^{-}\right.$ ${ }^{3}$ ) foi classificado nas faixas $\langle 2 ; 2,0-4,0$ e $\rangle$, correspondendo às classes de disponibilidade baixa, média e alta. $\mathrm{O}$ teor de $\mathrm{Mg}\left(\mathrm{cmol}_{\mathrm{c}} \mathrm{dm}^{-}\right.$ ${ }^{3}$ ) foi classificado nas faixas $\leq 0,5 ; 0,5-1,0$ e $>1,0$, obedecendo as classes de disponibilidade baixo, médio e alto. Para os teores de MOS utilizou-se as faixas $\leq 2,5 ; 2,6$ $5,0 \mathrm{e}>5,0$, correspondentes as classes baixo, médio e alto

\subsection{Análise de dados}

Os dados obtidos foram apresentados através de gráficos e submetidos a análise exploratória utilizando-se da Análise Numérica com medidas de posição (média) e de dispersão [(desvio padrão e coeficiente de variação)] (ZIMMERMANN, 2014).

\section{Resultados E DiscuSSÃo}

A partir do levantamento dos laudos de análise de solo e de acordo com o Manual de Calagem e Adubação para os Estados do Rio Grande do Sul e de Santa Catarina (CQFS- RS/SC, 2016), observa-se que 53\% dos solos encaminhados para análise durante o período apresentam $\mathrm{pH}$ com valores menores que 5,$5 ; 30 \%$ com valores entre $5,5-$ 5,$9 ; 10 \%$ com valores $\geq 6,5$ e, $7 \%$ com valores entre 6,0-6,4 (Figura 1a). O valor médio de $\mathrm{pH}$ foi de 5,4, com desvio padrão (DP) de 0,55 e coeficiente de variação $(\mathrm{CV})$ dos resultados de $10,1 \%$. Dos $53 \%$ dos laudos ( 78) com valores de $\mathrm{pH}$ menores que 5,5, 23\% têm valor muito baixo para $\mathrm{m} \%, 34 \%$ valores baixos, $13 \%$ valores médios, $23 \%$ valores altos e $7 \%$ apresentam valores muito altos (Figura 1b).

$\mathrm{O} \mathrm{pH}$ entre 2 e 3 indica presença de ácidos livres provenientes da pirita, $\mathrm{pH}$ entre 4 e 5 indica a presença de alumínio trocável $\left(\mathrm{Al}^{+3}\right)$ e $\mathrm{pH}$ em torno de 5,2 a 5,3 indica que o $\mathrm{Al}^{+3}$ está quase na sua totalidade insolubilizado e não causa mais danos as raízes, enquanto, o pH igual ou superior a 5,5 não apresenta mais o $\mathrm{Al}^{+3}$ (SOBRAL et al., 2015). A acidez do solo é um problema grave sendo prejudicial ao desenvolvimento das espécies vegetais, principalmente em sistemas de cultivo e, normalmente, associa-se a Vol. 18, N. 01 (2021) 

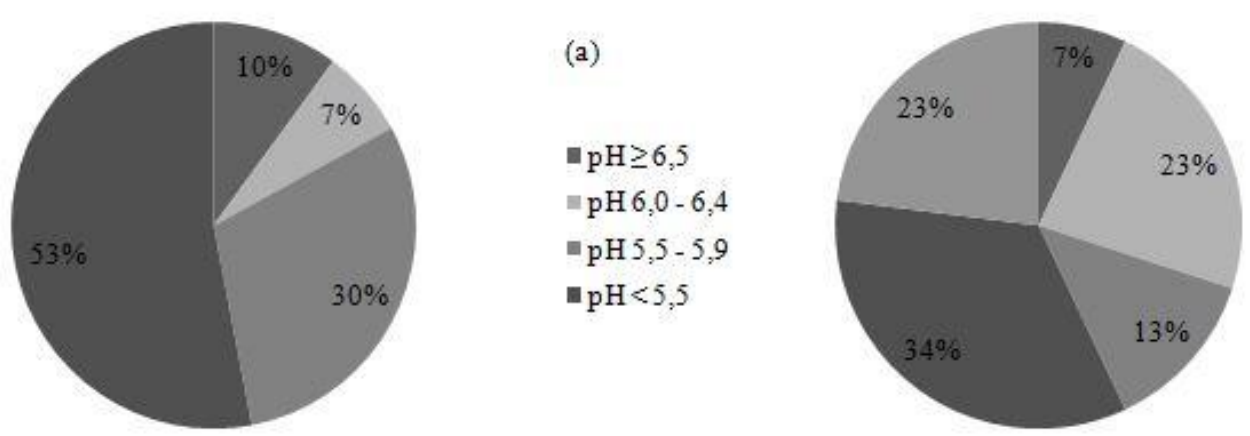

(b)

Muito alto: $>45 \%$

III Alto: $20,1-45 \%$

- Médio: 10,1-20\%

- Baixo: $5,0-10 \%$

Muito baixo: $<5 \%$

Figura 1. Distribuição de frequência do potencial hidrogeniônico ( $\mathrm{pH}$ ) (a) e saturação por alumínio (m\%) (b) dos laudos de análise de solo. Valores correspondentes a 147 propriedades rurais do município de Chapecó - SC, com amostras encaminhadas para análise básica no Laboratório de solos da Epagri - Chapecó, nos anos de 2016 e 2017.

presença de elementos como o $\mathrm{Al}^{+3}$ e $\mathrm{o}$ manganês $\left(\mathrm{Mn}^{+2}\right)$ em concentrações consideradas tóxicas, e de baixa disponibilidade de cátions de caráter básico como o $\mathrm{Ca}$ e $\mathrm{Mg}$. Quando em excesso, a acidez promove alterações na química e fertilidade do solo causando restrições ao crescimento radicular que incluem $o$ engrossamento das raízes e menor número de ramificações, o que limita a absorção de água e nutrientes afetando diretamente a produtividade das culturas (SOUSA et al., 2007).

Do total dos laudos que apresentam valores de $\mathrm{pH}$ menores de 5,5, 43\% apresentam valores de $\mathrm{m} \%$ medianamente prejudiciais ao desenvolvimento das espécies vegetais, sendo $30 \%$ destes considerados prejudiciais e altamente prejudiciais segundo Osaki (1981). Mesmo sendo um processo natural, a acidificação do solo pode ocorrer de forma acelerada em solos produtivos pela intensificação do uso, devido a lixiviação de cátions básicos causada pelas chuvas, absorção de nutrientes pelo sistema radicular das plantas e uso de fertilizantes nitrogenados. Por meio da análise periódica do solo, pode-se diminuir sua acidez pelo uso de calcário, evitando a perda de qualidade produtiva associada à acidificação (PROCHNOW e CANTARELLA, 2015). Desta forma, uma prática necessária as propriedades que tiveram o encaminhamento de análise neste período seria a realização da correção da acidez do solo para aumentar a resposta produtiva de suas culturas.

Não menos importante, devemos ainda chamar atenção para o fato de que da mesma forma que a acidez é um ponto limitante ao desenvolvimento das espécies, valores elevados de $\mathrm{pH}$ também são considerados preocupantes. Chamamos a atenção que aproximadamente $10 \%$ dos laudos apresentaram $\mathrm{pH}$ superior a 6,5, sendo que a partir destes valores o $\mathrm{pH}$ pode afetar a disponibilidade de micronutrientes por diminuir a solubilização e absorção de cobre $(\mathrm{Cu})$, zinco $(\mathrm{Zn})$, ferro $(\mathrm{Fe})$ e manganês $(\mathrm{Mn})$, e aumentar a disponibilidade de molibdênio (Mo) (DECHEN et al., 2018).

A distribuição de frequência para V\% (Figura 2) demonstra que 9\% dos laudos de solo apresentaram valores $\geq 85 \%, 47 \%$ valores de $65-74 \%, 39 \%$ valores de $75-85 \%$ e apenas $5 \%$ destes valores $<65 \%$.O valor médio de V\% encontrado nos laudos foi de $69 \%$ com DP e CV de 14 e $21 \%$, respectivamente. $\mathrm{O} \mathrm{V} \%$ é utilizado para o cálculo da necessidade de calcário e para se conhecer o nível de fertilidade no solo (SOBRAL et al., 2015) e indica quanto por cento dos pontos de troca de cátions nos coloides estão ocupados por bases ou, em outras palavras, quanto por cento das cargas negativas estão ocupadas por $\mathrm{Ca}, \mathrm{Mg}$ e $\mathrm{K}$, em relação aos pontos de troca dos cátions ácidos $\left(\mathrm{H}^{+} \mathrm{e} \mathrm{Al}{ }^{3+}\right)$ (GUARÇONI, 2017). 


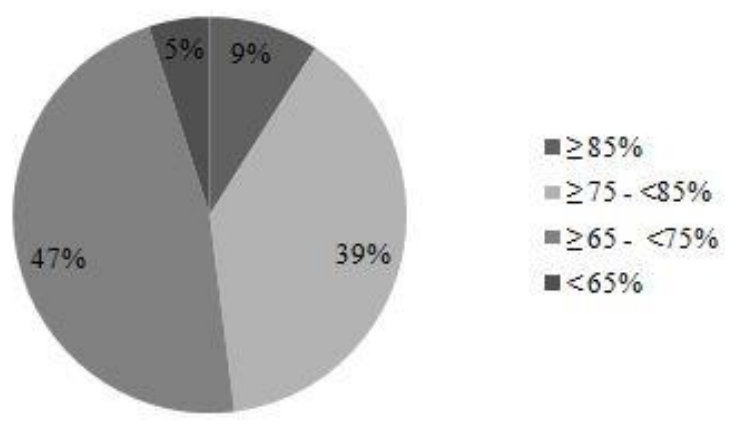

Figura 2. Distribuição de frequência da saturação de bases (V\%) dos laudos de análise de solo. Valores correspondentes a 147 propriedades rurais do município de Chapecó - SC, com amostras encaminhadas para análise básica no Laboratório de solos da Epagri - Chapecó, nos anos de 2016 e 2017.

Dos laudos avaliados $10 \%$ apresentam classe textural 1 (>60\% de argila), 5\% classe textural 2 (60 - 41\% de argila), $18 \%$ classe textural 3 (40 - 21\% de argila) e 67\% classe textural 4 ( $\leq 20 \%$ de argila) (Figura 3$)$. Desta maneira, a interpretação dos teores de $\mathrm{P}$ para os solos classe 1 (Figura 4a) mostra que 50\% apresentam disponibilidade muito alta, $11 \%$ alta, $18 \%$ média e $18 \%$ baixa, sendo que apenas 3\% apresentam disponibilidade muito baixa; enquanto, para os solos de classe 2, $27 \%$ apresentam disponibilidade muito alta, $22 \%$ alta, $17 \%$ média, $24 \%$ baixo e $10 \%$ muito baixa (Figura 4b). Para os solos de classe 3, $13 \%$ apresentam disponibilidade muito alta, $38 \%$ alta, $10 \%$ média, $24 \%$ baixa e $10 \%$ muito baixa (Figura 4c). Finalmente, para os solos de classe 4, 46\% mostram disponibilidade muito baixa, $21 \%$ baixa, $11 \%$ média, $13 \%$ alta e $9 \%$ muito alta (Figura 4d).

$\mathrm{O}$ valor médio obtido de $\mathrm{P}$ nos laudos foi de $23,9 \mathrm{mg} \mathrm{dm}^{-3}$, com valores altos de DP $(32,52)$ e CV $(136 \%)$.O intervalo de valores encontrados nos laudos variou de 0,8 a $177 \mathrm{mg}$ de $\mathrm{P} \mathrm{dm}^{-3}$, ambos na mesma classe textural de solo (classe 2), evidenciando a grande variabilidade do nutriente nos solos analisados, sendo responsável pelos altos valores de DP e CV encontrados para o nutriente.
Ao analisar as classes texturais (1,2 e 3) que representam solos do município de Chapecó de maior aptidão ao cultivo agrícola (Latossolo e Cambissolo) e maior capacidade de adsorção do nutriente, verifica-se que praticamente $50 \%$ destes solos e, em alguns casos acima desse valor, apresentam teores muito alto a alto de $\mathrm{P}$. Tal condição indica um manejo do nutriente de forma inadequada, com aplicações muito vezes em excesso e de forma desnecessária, principalmente na faixa de interpretação muito alta do nutriente, indicando a necessidade de moderação na aplicação desse nutriente nas lavouras. Outra possível resposta aos valores elevados de $\mathrm{P}$ encontrados nos solos amostrados refere-se a à aplicação de dejeto suíno no Oeste Catarinense, podendo causar o acúmulo deste nutriente no solo e causar poluição ambiental, trazendo efeitos negativos até mesmo para a produtividade das culturas (KLEIN e AGNE, 2012).

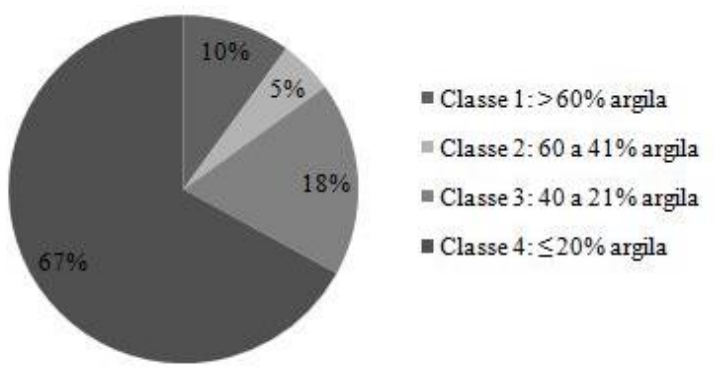

Figura 3. Distribuição de frequência da classe textural dos solos baseada nos teores de argila dos laudos de análise, utilizados para a interpretação dos teores de fósforo $(\mathrm{P})$ das amostras. Valores correspondentes a 147 propriedade rurais do município de Chapecó SC, com amostras encaminhadas para análise básica no Laboratório de solos da Epagri Chapecó, nos anos de 2016 e 2017.

Levantamento do Plano de Desenvolvimento Rural do Município (PREFEITURA MUNICIPAL DE CHAPECÓ, 2015) aponta que no ano de 2013 o número total de suínos contabilizado em Chapecó era de 60.773 animais provenientes de todas as fases de produção. $\mathrm{O}$ documento Vol. 18, N. 01 (2021) 
também menciona assim como um levantamento da qualidade das águas subterrâneas realizado por Facco et al. (2019), um elevado índice de contaminação das águas subterrâneas no interior do município com nitrato $\left(\mathrm{NO}_{3}{ }^{-}\right)$e atribuem a atividades como a criação intensiva de suínos e aves, bem como ao descarte incorreto dos dejetos em áreas agrícolas e outras práticas. Existe uma preocupação generalizada pelas quantidades de $\mathrm{N}$ e $\mathrm{P}$ originadas dos dejetos suínos que estão causando impactos ambientais, principalmente pela eutrofização das fontes de água. A aplicação de grandes quantidades do dejeto ao solo pode gerar excessos de nitratos, fósforo, salinização, presença de compostos xenobióticos (como agrotóxicos e produtos farmacêuticos), patógenos e emissões de gases de efeito estufa, e contaminação com micronutrientes $(\mathrm{Cu}, \mathrm{Zn}$ e $\mathrm{Mn}$ ) (MEURER et al., 2000).

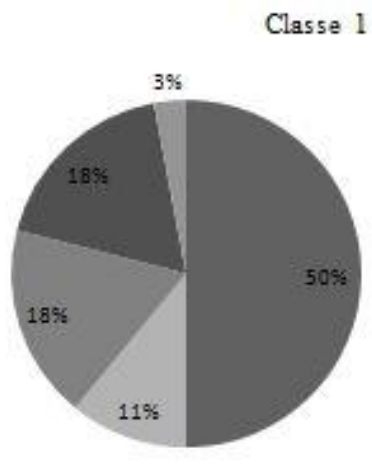

(a)

Muito alto: $>18,0$

Alto: $9,1-18,0$

Médio: $6,1-9,0$

- Baixo $3,1-6,0$

- Muito baixo $\leq 3,0$

Classe 3

(c)

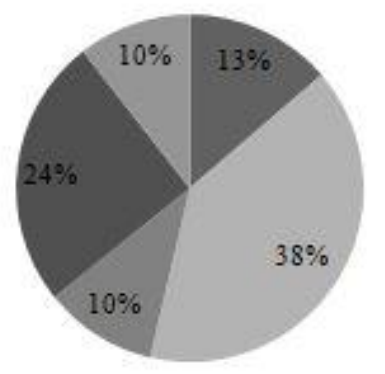

$=$ Muito alto: $>36,0$

Alto: $18,1-36,0$

- Médio: 12,1 - 18,0

Baixo: $6,1-12,0$

Muito baixo: $\leq 6,0$
De forma contrária, os solos de menor capacidade de adsorção e menor aptidão agrícola, como os da classe textural 4, apresentam na sua maioria valores muito baixo e baixo de P (Figura 4d). Muitas vezes estes solos, embora também possam ser utilizados em algumas propriedades do município para a produção de grãos, são na grande maioria utilizados para a produção animal e demonstram muitas vezes a despreocupação do produtor em realizar adubação de suas pastagens.

Dos laudos analisados 69\% apresentaram valor de $\mathrm{CTC}_{\mathrm{pH} 7,0}\left(\mathrm{cmol}_{\mathrm{c}} \mathrm{dm}^{-3}\right)$ média, $29 \%$ alta e o restante $2 \%$ (1\% para cada faixa) enquadra-se como muito alta e baixa (Figura 5). Esses valores indicam grande capacidade de reserva de nutrientes para as plantas na grande maioria dos solos amostrados e corroboram com os resultados encontrados por Mafra et al. (2011) que, ao

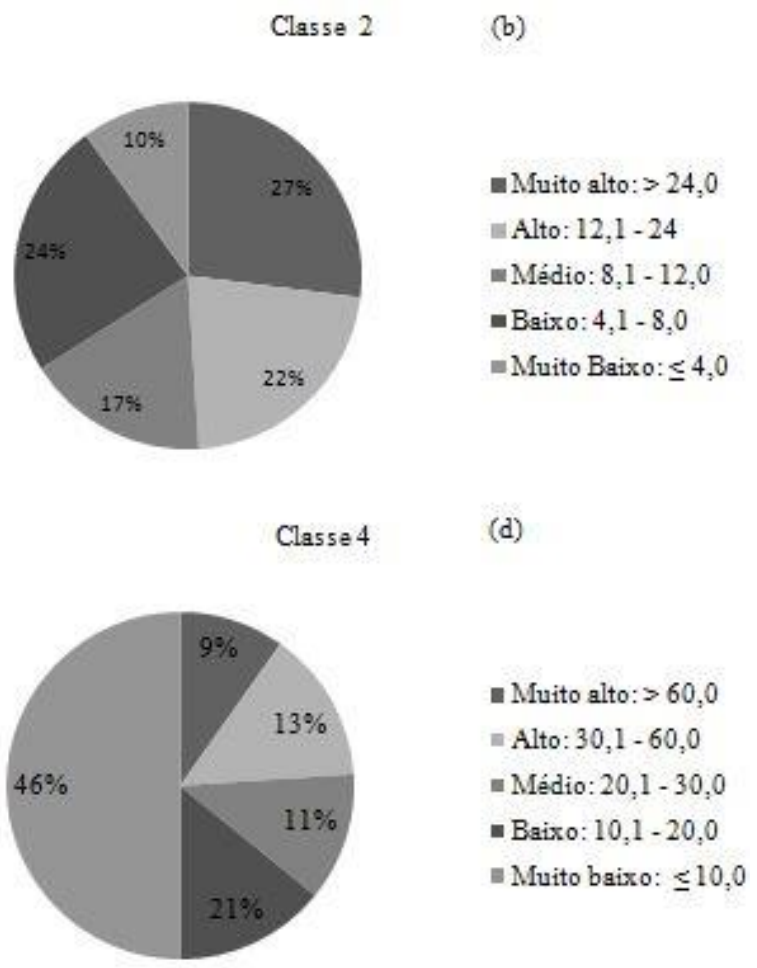

Figura 4. Distribuição de frequência dos teores de fósforo $(\mathrm{P})\left(\mathrm{mg} \mathrm{dm}^{-3}\right)$ dos laudos de análise de solo, dentro das classes de teor de argila. Valores correspondentes a147 propriedades rurais do município de Chapecó - SC, com amostras encaminhadas para análise básica no Laboratório de solos da Epagri - Chapecó, nos anos de 2016 e 2017. 
estudarem solos de vinícolas na Serra Catarinense, obtiveram média de 13,9 $\mathrm{cmol}_{\mathrm{c}}$ $\mathrm{dm}^{-3}$, caracterizando uma CTC de faixa média. Para o presente estudo, a CTC média foi de $13,7 \mathrm{cmol}_{\mathrm{c}} \mathrm{dm}^{-}{ }^{3}$, o DP de 4,1 e o CV de $30,1 \%$, com intervalo de 3,28 a $31,7 \mathrm{cmol}_{\mathrm{c}} \mathrm{dm}^{-}$ 3 .

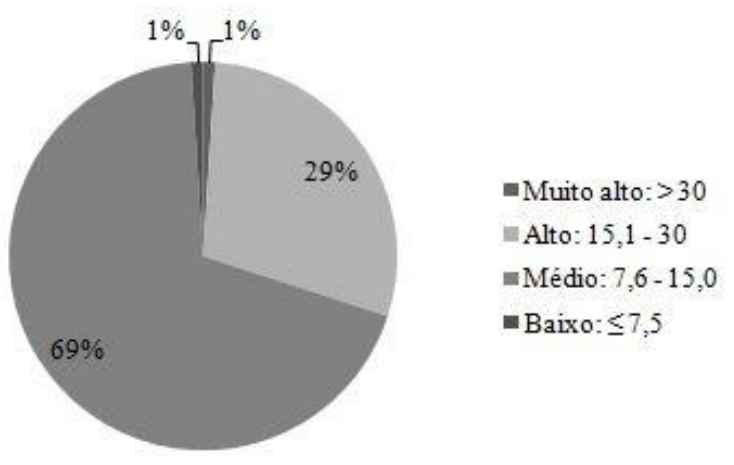

Figura 5. Distribuição da frequência da capacidade de troca de cátions $\left(\mathrm{CTC}_{\mathrm{pH}} 7,0\right.$, $\mathrm{cmol}_{\mathrm{c}} \mathrm{dm}^{-3}$ ) dos laudos de análise do solo. Valores correspondentes a 147 propriedades rurais do município de Chapecó- SC, com amostras encaminhadas para análise básica no Laboratório de solos da Epagri - Chapecó, nos anos de 2016 e 2017.

Considerando-se a CTC dos laudos avaliados, utilizou-se a tabela 6.9 (p. 96) do Manual de Adubação e Calagem para os Estados do Rio Grande do Sul e Santa Catarina (CQFS- RS/SC, 2016) para a interpretação da classe de disponibilidade de potássio (K). Na CTC de maior frequência de ocorrência (classe média, faixa entre 7,6 a 15 $\left.\mathrm{cmol}_{\mathrm{c}} \mathrm{dm}^{-3}\right) 100 \%$ dos laudos avaliados têm os níveis de $\mathrm{K}\left(\mathrm{mg} \mathrm{dm}^{-3}\right)$ classificados como muito baixo (Figura 6a); na CTC alta (15,1 a $30 \mathrm{cmol}_{\mathrm{c}} \mathrm{dm}^{-3}$ ) $61 \%$ das amostras classificamse como muito alto, $24 \%$ como alto e $15 \%$ como média (Figura 6b). Na classe de CTC muito alta $\left(>30,0 \mathrm{cmol}_{\mathrm{c}} \mathrm{dm}^{-3}\right) 100 \%$ dos laudos classificam-se em alto (Figura 6c); enquanto, na classe de CTC baixa $(\leq 7,5$ $\mathrm{cmol}_{\mathrm{c}} \mathrm{dm}^{3}{ }^{3}$ ) os valores de $\mathrm{K}$ classificam-se em alto $(67 \%)$ e muito alto (33\%) (Figura 6d). O valor médio de $\mathrm{K}$ foi de $186 \mathrm{cmol}_{\mathrm{c}} \mathrm{dm}^{-3}$, com intervalo de 12 a $560 \mathrm{cmol}_{\mathrm{c}} \mathrm{dm}^{-3}$, DP de 113,5 e CV de $61,2 \%$.

Os dados apontam que na CTC de maior ocorrência encontrada nos laudos (CTC média) a alta frequência de propriedades que apresentam teores muito baixo de $\mathrm{K}$ no solo pode estar associada a um manejo com baixa presença de palhada no solo. $\mathrm{O}$ cultivo de milho para silagem, atividade muito difundida no Oeste Catarinense e, também no município de Chapecó, é um exemplo de prática com baixa manutenção de material superficial. Neste tipo de cultivo, o processo de colheita faz a completa retirada da planta da lavoura, deixando o solo sem material orgânico de cobertura, favorecendo a exportação de nutrientes no solo, principalmente $\mathrm{o} K$ (MELO et al., 2019). Além disso, baixos valores de $\mathrm{pH}$, como no caso da avaliação das amostras de Chapecó, aumentam a lixiviação de $\mathrm{K}$ até mesmo em solos mais argilosos (Prado e Natali, 2004), podendo ser ainda maior esta lixiviação quando da prática de gessagem (prática também crescente no município) em solos não corrigidos (SOUZA et al., 2012).

Por outro lado, valores elevados do nutriente em solos de CTC maior podem mostrar justamente o contrário, indicando o uso excessivo da adubação potássica e/ou um manejo que favoreça a manutenção de resíduos vegetais, uma vez que o sistema plantio direto (SPD) é o manejo dominante do município. $\mathrm{O} K$ não se liga a compostos orgânicos e fica disponível sob sua forma iônica no citoplasma, facilitando a sua rápida disponibilização no solo quando em sistemas que incorporem palha como o plantio direto (MEURER et al., 2018), o que favorece o aumento dos teores deste nutriente em lavouras sobre este sistema, aumentando com o período de implantação do SPD. 


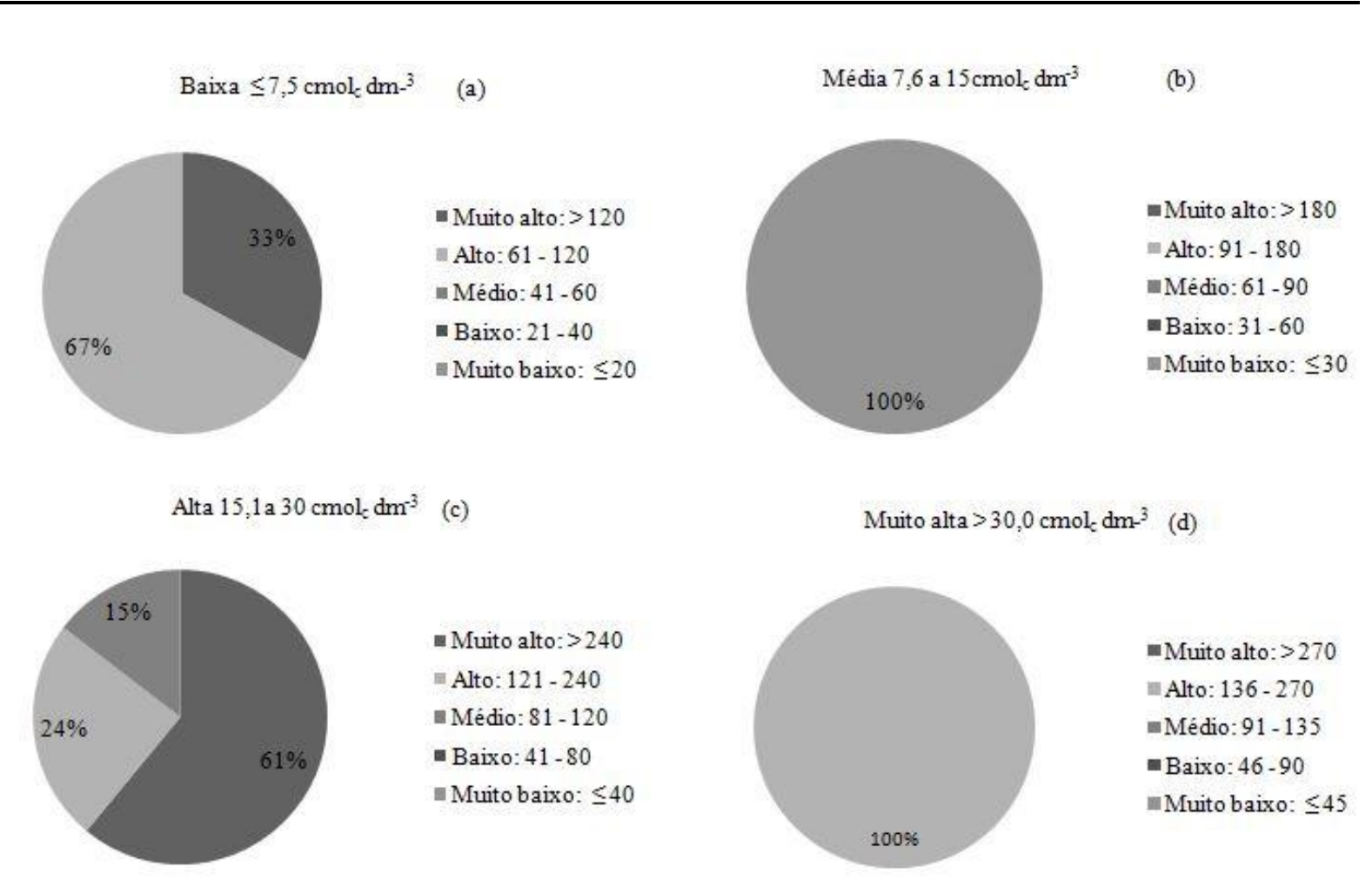

Figura 6. Distribuição da frequência do teor de potássio $(\mathrm{K})\left(\mathrm{mg} \mathrm{dm}^{-3}\right)$ dos laudos de análise de solo conforme a CTC do solo $\left[\leq 7,5 ; 7,6\right.$ a 15,0; 15,1 a 30,0 e $>30 \mathrm{cmol}_{\mathrm{c}} \mathrm{dm}^{-3}$, respectivamente baixa (a), média (b), alta (c) e muito alta (d)].Valores correspondentes a 147 propriedades rurais do município de Chapecó - SC, com amostras encaminhadas para análise básica no Laboratório de solos da Epagri - Chapecó, nos anos de 2016 e 2017.

Para os teores de cálcio $(\mathrm{Ca})$ e magnésio (Mg) dos laudos analisados, a maior frequência ocorreu na classe de disponibilidade alta para ambos os nutrientes, respectivamente, 86 e 93\% (Figuras 7a,b). O valor médio de $\mathrm{Ca}$ obtido nas amostras foi de 6,7 $\mathrm{cmol}_{\mathrm{c}} \mathrm{dm}^{-3} \mathrm{com}$ DP de 3,3 e CV de 48,5\%, com intervalo de 0,1 a $21,4 \mathrm{cmol}_{\mathrm{c}} \mathrm{dm}^{-3}$. Para o $\mathrm{Mg}$ o valor médio obtido nas amostras foi de 2,4 $\mathrm{cmol}_{\mathrm{c}} \mathrm{dm}^{-3}$ com DP de 1,3 e CV de $52 \%$, com intervalo de 0,1 a $8,8 \mathrm{cmol}_{\mathrm{c}} \mathrm{dm}^{-3}$. Esses valores podem ser comparados com os resultados encontrados por Groth et al. (2013), que estudando os solos de São José do Cedro - SC, encontraram valores semelhantes aos solos de Chapecó para os teores de $\mathrm{Ca}$ e $\mathrm{Mg}$, sendo que os mesmos se assemelham quanto a classificação, clima e práticas de manejo predominantes nos municípios. Sistemas de cultivo como o cultivo mínimo, prática que assim como o plantio direto é adotada por produtores da região de Chapecó, principalmente os que associam a produção de grãos e pastagens, realizando o que muitos denominam como escarificação "leve" para semeadura de pastagens de inverno, afeta a distribuição dos nutrientes no perfil do solo, ocorrendo maior concentração dos elementos de menor mobilidade na solução nas camadas superficiais

(PROCHNOW CANTARELLA, 2015). No entanto, os valores altos encontrados para o $\mathrm{Ca}$ mesmo em valores de $\mathrm{pH}$ mais baixo (78 laudos com $\mathrm{pH}<5,5 ; 126$ laudos do total de $147 \mathrm{com}$ valores altos de $\mathrm{Ca}$ ), nos levam a um possível alerta quanto a uma prática crescente no Oeste Catarinense - a aplicação muitas vezes sem critério agronômico para a utilização do gesso agrícola $\left(\mathrm{CaSO}_{4} \cdot 2 \mathrm{H}_{2} \mathrm{O}\right)$. Em alguns casos, o gesso pode sim ser utilizado para corrigir os problemas de excesso de $\mathrm{Al}$ e falta de $\mathrm{Ca}$ no subsolo, permitindo o crescimento radicular profundo, o que é importante para a absorção de água e nutrientes abaixo das camadas 
superficiais do solo (PROCHNOW e CANTARELLA, 2015); porém, não substitui o calcário para correção das camadas superficiais.

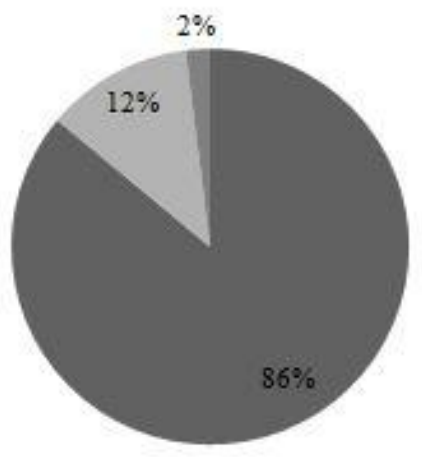

(a)

Alto: $>4$

Médio: $2,0-4,0$

- Baix $0:<2$ teores de matéria orgânica do solo (MOS, \%) dos laudos de análise de solo. Valores correspondentes a 147 propriedades rurais do município de Chapecó - SC, com amostras

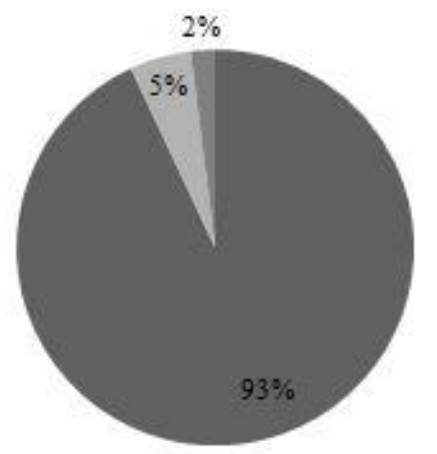

Alto: $>1$

Médio: $0,5-1,0$

- Baixo: $<0,5$

Figura 7. Distribuição da frequência dos teores de cálcio $\left(\mathrm{Ca}, \mathrm{cmol}_{\mathrm{c}} \mathrm{kg}^{-1}\right)$ (a) e magnésio $(\mathrm{Mg}$, $\mathrm{cmol}_{\mathrm{c}} \mathrm{kg}^{-1}$ ) (7) dos laudos de análise de solo. Valores correspondentes a 147 de propriedades rurais do município de Chapecó - SC, com amostras encaminhadas para análise básica no Laboratório de solos da Epagri - Chapecó, nos anos de 2016 e 2017.

O gesso muitas vezes é confundido com corretivo de solo pelo produtor que o aplica achando que irá promover alterações do $\mathrm{pH}$ em superfície e aumento dos teores de $\mathrm{Ca}$ no solo (PROCHNOW, 2014). Tal condicionador do solo, principalmente em solos não corrigidos, pode promover $\mathrm{o}$ deslocamento do $\mathrm{K}$ dos sítios de ligação, promovendo o seu deslocamento para a solução do solo e causando a lixiviação desse nutriente (BARTOLO et al., 2017). Essa explicação pode ser uma das causas dos baixos teores de K (69\% dos laudos) encontrados nas amostras do município (Figura 6a), associado a outras possíveis causas já mencionadas anteriormente.

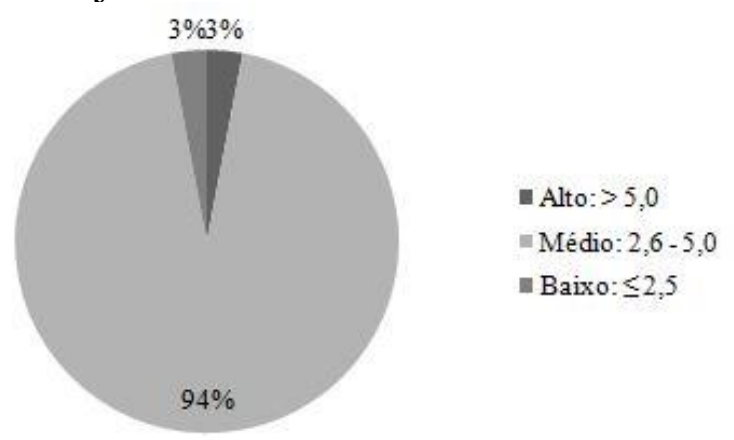

Figura 8. Distribuição da frequência dos encaminhadas para análise básica no Laboratório de solos da Epagri - Chapecó, nos anos de 2016 e 2017.

$\mathrm{O} \mathrm{Mg}$ se destaca por ser um ativador das enzimas relacionadas com o metabolismo energético, além de servir de ligação entre as estruturas de pirofosfato do ATP (adenosina trifosfato) e ADP (adenosina difosfato) (COELHO et al., 2012). A maior frequência de amostras com elevado teor deste nutriente (137 do total de 147) pode ser o resultado da formação geológica do município, que tem o basalto, uma rocha extrusiva ferromagnesiana, disponibilizando $\mathrm{Mg}$ para o solo quando do seu intemperismo (NARDY et al., 2008).

Quanto ao conteúdo de MOS dos laudos analisados (Figura 8), 94\% destes apresentaram teores médios de MOS, sendo apenas $3 \%$ alto e $3 \%$ baixo. $\mathrm{O}$ valor médio obtido nas amostras foi de 3,5\% com DP de 0,7 e CV de 20,8\%, com intervalo de 1,6 a7\%, respectivamente. Estes valores médios podem ser comparados com os valores encontrados por Groth et al. (2013), no município de São José do Cedro, próximo a Chapecó. A MOS mesmo representando uma pequena fração da 
fase sólida de solos tropicais, apresenta papel importante na manutenção da qualidade física, química e biológica, podendo contribuir de 20 - 90\% da CTC das camadas superficiais de solo (SILVA e MENDONÇA, 2007). Práticas que favoreçam a conservação da MOS como o SPD - prática de manejo dominante no município de Chapecó, melhoram as propriedades do solo e ajudam a reduzir o risco de erosão; principalmente em solos de declividade acentuada como os do município.

\section{CONSIDERaÇões FINAIS E Algumas Recomendações TÉCNICAS}

Considerado um município de vocação agrícola, um diagnóstico inicial da fertilidade dos solos de Chapecó pode fornecer caminhos que direcionem a melhoria da assistência técnica voltada à recuperação e/ou manutenção da fertilidade do solo. Segundo dados preliminares do IBGE (2018) o município apresenta 1.661 propriedades, 176 propriedades a menos do que aponta o Plano de Desenvolvimento Rural do Município de Chapecó publicado no ano 2015. Deste total, no período do levantamento dos dados aqui apresentados, apenas 147 propriedades encaminharam amostras para análise, representando apenas $8,8 \%$ deste total. Embora saibamos que parte das análises sejam encaminhadas via cooperativas e não necessariamente pela assistência técnica da Epagri ou produtor, a própria cooperativa do município apresenta convênio junto a Epagri para as análises, e do total encaminhado naqueles anos computa-se as realizadas pela Cooperativa.

No Plano de Desenvolvimento Rural do Munícipio de Chapecó (PREFEITURA DE CHAPECÓ, 2018), menciona-se que a conservação dos solos deve ser uma preocupação do poder público municipal e dos produtores rurais, de forma individual e coletiva, sendo necessário o estabelecimento de metas com vistas à manutenção e/ou recuperação das boas condições das terras, estradas e unidades de conservação. Uma orientação para a construção destas metas envolveria um comprometimento técnico ao estímulo para a necessidade de aumentar o número de análises realizadas nas propriedades do município, bem com o aumento da frequência da realização das mesmas. A análise de solo constitui de ferramenta confiável para a determinação da fertilidade atual de determinada área, representando também parte importante do planejamento da instalação e manutenção de culturas agrícolas. Justamente através deste conhecimento se torna possível o uso de práticas de manejo como o uso de corretivos, fertilizantes e gessagem, de forma racional e direcionada para a real necessidade da área.

$\mathrm{Na}$ maioria dos solos avaliados, os atributos de fertilidade apresentaram valores com grande oscilação entre o mínimo e máximo encontrado, sendo responsáveis pela grande variabilidade média dos nutrientes verificada pelos desvios padrões e coeficiente de variação dos mesmos. Os valores médios de $\mathrm{K}$ encontrados na grande maioria dos laudos avaliados (69\% do total) chama a atenção para manejos com baixa manutenção de palhada, elevada exportação do nutriente das lavouras - como no caso de cultivos para silagem comuns também no município, e até mesmo mecanismos de lixiviação do nutriente. Desta forma, recomenda-se que as Cooperativas, Secretaria de Agricultura e Epagri, possam olhar de maneira mais cuidadosa para essas condições de maneira pontual nas propriedades do município.

Da mesma maneira, concentrações excessivas para alguns nutrientes como o $\mathrm{Ca}$, $\mathrm{Mg}$ e $\mathrm{P}$, e até mesmo o $\mathrm{K}$ em algumas classes de CTC, mostra haver variabilidade de solos e manejo dos nutrientes, com aplicações de fertilizantes sem levar em conta os resultados da análise de solo e a real necessidade da cultura - prática impensável na agricultura atual, devendo ser acompanhado de perto pela assistência técnica local. Se o produtor não realiza a análise de solo de maneira frequente 
e de forma sistemática, as recomendações de adubações e corretivos irão subestimar ou superestimar a quantidade adequada, levando a contaminação ambiental e maior custo das lavouras.

Outro aspecto a se considerar é que a maioria significativa dos solos do município (acima de 50\%) apresentam seu $\mathrm{pH}<5,5$, mostrando uma negligência por parte do produtor quanto à correção do solo que, consequentemente, afeta a produtividade de suas áreas. Além disso, os valores altos de $\mathrm{Ca}$ encontrados na maioria dos laudos que apresentavam o $\mathrm{pH}$ baixo devem orientar a assistência técnica para a busca da real causa destes resultados, para que o desequilíbrio de nutrientes não afete os cultivos agrícolas.

Portanto, o estudo mostra que os parâmetros químicos do solo podem ser usados para a predição da qualidade do mesmo e do manejo a ser adotado por propriedades rurais do município de Chapecó - SC, chamando atenção a orientações de práticas como calagem, correta aplicação do gesso, melhoria do manejo da palhada em áreas de baixa disponibilidade de $K$, orientações quanto ao cultivo de milho silagem e diminuição da adubação fosfatada.

\section{Agradecimentos}

Os autores agradecem ao Laboratório de Solos da Empresa de Pesquisa Agropecuária e Extensão Rural de Santa Catarina - EPAGRI/CEPAF/CHAPECÓ pela disponibilização dos laudos para realização do estudo.

\section{REFERÊNCIAS}

ANJOS DC, HERNANDEZ FF,COSTA JM, CABALLERO SU, MOREIRA VO. G. Fertilidade do solo, crescimento e qualidade de frutos do mamoeiro Tainung sob fertirrigação com potássio. Revista Ciência Agronômica, v. 46, n. 4, p. 774-785, 2015.
ARAÚJO EA, KER JC, NEVES JL, LANI JL. Qualidade do solo: Conceitos, indicadores e avaliação. Revista Brasileira Aplicada nas Ciências Agrária, Guarapuava, v.5, n.1, p.187-206, 2012.

BALDOTTO MA, VIEIRA EM, SOUZA DO, BALDOTTO LB. Estoque e frações de carbono orgânico e fertilidade de solo sob floresta, agricultura e pecuária. Revista Ceres, Viçosa, v. 62, n. 3, p. 301-309, 2015.

BARTOLO I, FERNANDES R, SEMPITERNO C. Valorização agrícola de lamas: disponibilidade do fósforo. Revista de Ciências Agrárias, Lisboa, v. 40, n. 3, p. 497 505, 2017.

BATISTA TE, NÓBREGA MP, JÚNIOR AA, MOREIRA PS, MARACAJÁ PB. Indicadores de qualidade do solo em agroecossistemas. Revista Brasileira de Gestão Ambiental, Pombal, v.7, n. 1, p.102129, 2013.

BISSANI CA. GIANELLO C. CAMARGO FAO, TEDESCO, M.J. Fertilidade dos solos e manejo da adubação de culturas. 2. ed. Porto Alegre: Metrópole, p. 344.2008.

COELHO VT, RODAS CL, COELHO LC, CARVALHO JG, ALMEIDA EA, FIGUEIREDO MV. Caracterização de sintomas visuais de deficiência de macronutrientes e boro em planta de gengibre ornamental. Revista Brasileira de Horticultura Ornamental, v.18, n.1, p.47$55,2012$.

COMISSÃO DE QUÍMICA E FERTILIDADE DO SOLO - RS/SC. Manual 
de calagem e adubação para os Estados do Rio Grande do Sul e Santa Catarina. 11. ed. Porto Alegre; Sociedade Brasileira de Ciência do Solo, Núcleo Regional Sul. UFRGS, 2016.

CREPALDI RA, PORTILHO IR, SILVESTRE R, MERCANTE FM. Formigas como bioindicadores de qualidade do solo no sistema integrado lavoura-pecuária. Ciência Rural, Santa Maria, v. 44, n. 5, p. 781-787, 2014.

FACCO J, CARASEK FL, OLIVEIRA JUNIOR SF, SCHEIBE LF, PASSOS MG dos, BLANK MM. Qualidade das águas subterrâneas no Oeste de Santa Catarina, Brasil. In: AGUILERA JG, ZUFFO AM. Ciências Exatas e da Terra e a Dimensão Adquirida através da Evolução Tecnológica. 2 ed. Ponta Grossa, PR: Atena Editora, 2019. Cap. 17, p. 182.

FERNANDES MS, SOUZA SR, SANTOS LA. Nutrição Mineral de plantas. In: DECHEN AR, NACHTIGALL GR, CARMELLO QAC, SANTOS LA, SPERANDIO ML. Micronutrientes. 2 ed. Viçosa, MG: Sociedade Brasileira de Ciências do Solo, 2018. Cap. 14, p 491.

FERNANDES MS, SOUZA SR, SANTOS LA. Nutrição Mineral de plantas. In: MEURER EJ, TIECHER T, MATTIELLO L. Potássio. 2 ed. Viçosa, MG: Sociedade Brasileira de Ciências do Solo, 2018. Cap. 12, p 429.

GROTH MZ, ROZA-GOMES MF, LAJÚS CR. Avaliação da fertilidade do solo no município de São José do Cedro, SC, Brasil. Evidência, Joaçaba v. 13, n. 2, p. 109-122, 2013.
GUARÇONI A. Saturação por bases para o cafeeiro baseada no $\mathrm{pH}$ do solo e no suprimento de $\mathrm{Ca}$ e $\mathrm{Mg}$. Coffee Science, Lavras, v.12, n.3, p. 327-336, 2017.

INSTITUTO BRASILEIRO DE GEOGRAFIA E ESTATISTICA - IBGE. Censo agropecuário. Rio de Janeiro, 2018. Disponível em: <https://cidades.ibge.gov.br/brasil/sc/chapeco /panorama>. Acesso em: 16 out. 2018.

KLEIN C, AGNE SA. Fósforo: de nutriente à poluente. Revista Eletrônica em Gestão, Educação e Tecnologia Ambiental, Santa Maria, v. 8, n.8, p. 1713-1721, 2012.

MAFRA MH, CASSOL PC, MIQUELLUTI DJ, ERNANI PR, GATIBONI LC, FERREIRA EZ, BARROS M, ZALAMENA J, GROHSKOP MA. Atributos químicos do solo e estado nutricional de videira Cabernet Sauvignon (Vitis vinifera L.) na Serra Catarinense. Revista de Ciências Agroveterinárias, Lages, v. 10, n. 1, p. 44-53, 2011.

MELO D, PEREIRA LH, MAGGI MF, GMOATTO E, MARCHETTI I. Soil compaction in areas of maize used for silage with the application of wastewater. Revista de Ciência Agronômica, Fortaleza,v. 50, n. 2, p. 205-215.2019.

MEURER EJ, BISSANI C, SELBACH PA. Poluentes do solo e do ambiente. In: MEURER, E.J. (Ed.). Fundamentos de química do solo. 2 ed Genesis, Porto Alegre, 2000, p. 151-169. Fundamentos de química do solo. 2.ed. Porto Alegre, Gênesis, 2004. p.207-237. 
MEURER EJ, TIECHER T, MATTIELLO L. Potássio. Viçosa: CUBO, 2018. 670 p.

NARDY AR, MACHADO FB, OLIVEIRA MF. As rochas vulcânicas mesozóicas ácidas da Bacia do Paraná: Litoestratigrafia e considerações geoquímico - estratigráficas. Revista Brasileira de Geociências, v. 38, n. 1, p.178-195, 2008.

NOVAIS RF. Fertilidade do solo. In: SILVA IR, MENDONÇA ES. Matéria orgânica do solo.22 ed. Viçosa, MG: Sociedade Brasileira de Ciências do Solo, 2007. Cap. 6, p. 275.

NOVAIS RF. Fertilidade do solo. In: SOUSA DMG, MIRANDA LN, OLIVEIRA SA. Acidez do solo e sua correção. 22 ed. Viçosa, MG: Sociedade Brasileira de Ciências do Solo, 2007. Cap. 5, p. 205.

OLIVEIRA PR, DEMINICIS BB, CASTAGNARA DD, GOMES FN. Efeito da adubação com fósforo do capim Mombaça em solos com texturas arenosa e argilosa. Archivos de Zootecnia, Córdoba, v. 61, n. 235, p. 397406, 2012.

OSAKI, F. Calagem e adubação. Campinas: Instituto Brasileiro de Ensino Agrícola, 1991. $503 \mathrm{p}$.

PRADO RM, NATALE W. Calagem na nutrição de cálcio e no desenvolvimento do sistema radicular da goiabeira. Pesquisa Agropecuária Brasileira, Brasília, v. 39, n. 10, p. 1007- 1012, 2004.

PREFEITURA MUNICIPAL DE CHAPECÓ. Plano de desenvolvimento rural de Chapecó. 2015. Disponível em: <https://web.chapeco.sc.gov.br/document os/?f=/Sedema/Plano\%20de\%20Desenvolvi mento\%20Rural.pdf $>$. Acesso em: 30 out. 2018 .

PREZOTTI LC, GUARÇONI A. Guia de interpretação de análise de solo e foliar. Vitória: INCAPER, 2013. 104 p.

PROCHNOW LI, CARTARELLA, H. Manejo do solo visando o aumento da produtividade. Campinas: Informações Agronômicas, n. 151. 2015.

PROCHNOW LI. Avaliação do manejo e acidez do solo. Piracicaba: Informações Agronômicas, 2014.

SECRETARIA DE ESTADO DO PLANEJAMNETO, ORÇAMENTO E GESTÃO. Caracterização Regional. 2003. Disponível em:<file:///C:/Users/Usu\%C3\%A1rio/Downl oads/CHAPECO.pdf>. Acesso em: 25 Jun. 2020.

SOBRAL LF, BARRETTO MV, SILVA AJ, ANJOS JL. Guia prático para interpretação de resultados de análises de solo. 1.ed. Aracaju: Embrapa Tabuleiros Costeiros, v.1, p. $7-12,2015$.

SOUZA FR, JUNIOR EJR, FIETZ CR, BERGAMIN AC, ROSA YBJR, ZEVIANI WM. Efeito do gesso nas propriedades químicas do solo sob dois sistemas de manejo. Semina: Ciências Agrárias, Londrina, v. 33, n. 5 , p. $1717-1732,2012$ 
VARASCHINI AC. Avaliação da fertilidade do solo na agricultura de precisão. 2012. 55

p. Monografia (Bacharel em Agronomia) UNIJUÍ. IJUIí, 2012.

ZIMMERMANN FJP. Estatística aplicada à pesquisa agrícola. 2. ed. Brasília - DF: Embrapa, p. 582, 2014. 